\title{
Rats with Fimbria-Fornix Lesions Display a Place Response in a Swimming Pool: A Dissociation Between Getting There and Knowing Where
}

\author{
Ian Q. Whishaw, ${ }^{1}$ Jean-Christophe Cassel, ${ }^{2}$ and Leonard E. Jarrard ${ }^{3}$ \\ ${ }^{1}$ Department of Psychology, University of Lethbridge, Lethbridge, Alberta, Canada T1K 3M4, ${ }^{2} \mathrm{CNRS}$, UPR 419, \\ Université Louis Pasteur, Strasbourg, France, and '3Department of Psychology, Washington and Lee University, \\ Lexington, Virginia
}

Some theories of hippocampal formation function postulate that it is involved in using the relationships between distal cues for spatial navigation. That rats with damage to the hippocampal formation are impaired in learning place responses of escaping to a platform hidden just below the surface of the water of a swimming pool, supports this view. Using rats with fimbria-fornix (FF) lesions, we examined whether their impairment is related to an inability to learn how to reach the platform as opposed to learning its location. In a first experiment, the FF rats were impaired in learning to swim to a hidden platform but could swim to a visible platform. In a second experiment, after being pretrained to swim to a visible platform, the FF rats swam to, paused, and searched the vicinity of the platform's previous location when it was removed. This finding showed that the FF rats expected to find the platform at that location. Additional tests confirmed that they had learned a place response. Despite having acquired a place response, they still could not acquire new place responses when only the hidden platform training procedure was used. Thus, these results in dissociating the processes of "getting there" and "knowing where" suggest that the FF rats' impairment may be in some process of motoric control, such as path Integration, rather than in learning the location of the platform in relation to ambient cues. The results are discussed in relation to relevant theories of hippocampal function.

[Key words: configural learning and space, fimbria-fornix and space, hippocampus and space, path integration, place learning, rat spatial learning, spatial navigation]

A number of theories of hippocampal function suggest that it has a role in using the relations between ambient cues to guide movements (Hirsh, 1974; O'Keefe and Nadel, 1978; Sutherland and Rudy, 1989; Jarrard, 1993). In an exemplar task, rats placed in a swimming pool, in which a small escape platform is hidden just beneath the water's surface, will learn to swim directly to

\footnotetext{
Received Jan. 12, 1995; revised Mar. 30, 1995; accepted Apr. 6, 1995.

This research was supported by a grant from the Natural Sciences and Engineering Research Council of Canada, by a fellowship from Ministere de la Recherche et de la Technologie to I.Q.W., and grants from the Direction des Recherches et Etudes Techniques (93-086). We thank Joanne Tomie for assistance.

Correspondence should be addressed to Dr. Ian $\mathrm{Q}$. Whishaw at the above address.

Copyright (C) 1995 Society for Neuroscience $0270-6474 / 95 / 155779-10 \$ 05.00 / 0$
}

the platform from any location on the pool's edge (Morris, 1981). Rats with hippocampal dysfunction, produced by hippocampal removal (Morris et al., 1982; Sutherland et al., 1982a; Whishaw, 1987), damage to its efferents and afferents in the fimbria-fornix (Cassel and Kelche, 1989; Sutherland and Rodriguez, 1989), or cholinergic blockade (Sutherland et al., 1982b; Whishaw, 1985a,b; Whishaw and Tomie, 1987), are severely impaired. Since the same studies show that the rats remain excellent swimmers and can quickly learn to escape to a visible platform, the results suggest that the hippocampus is selectively involved in using the relational properties of ambient cues to guide movements.

Despite evidence that disruption of hippocampal function impairs spatial behavior, it is difficult to demonstrate that this is due to an inability to use the relations between ambient cues. As first demonstrated by Krechevsky (1938), problem solving involves at least two processes: discovering the task's solution and then learning the task. Similarly, in swimming pool tasks, animals must first learn to swim, discover that there is an escape, and find that effective guidance requires distal cues (Sutherland and Dyck, 1984; Whishaw and Petrie, 1988). In fact, during the first swimming trials, the rats engage in a variety of behaviors such as scrabbling at the edge of the pool, swimming around its edge, making sorties into the center of the pool, swimming in circles, etc., suggesting that they are searching for an appropriate solution. Once a solution is found, however, new place responses, even in tests in novel locations, can be acquired within one trial (Whishaw, 1985c, 1989). This raises the question of whether retarded place learning following hippocampal damage resides in finding and using an appropriate solution or "getting there," rather than, or in addition to, loss of the ability to know a location using ambient cues or "knowing where."

The present study addresses this question by demonstrating in a first experiment that rats with fimbria-fornix (FF) lesions are impaired in acquiring a place response. Then in a second experiment, FF rats are trained with a visible cue using a gradual adaptation procedure to determine whether first having been provided with an acquisition strategy they demonstrate subsequently that have acquired a place response as do control rats (Whishaw and Mittleman, 1986). Behavior is then assessed on probe trials with kinematic measures of swim direction, distance, and velocity, to determine whether the rats expected to find a platform in the pool and, more importantly, to determine whether they expect to find it at its previous location. In addition to this central 
experiment, control studies were performed to characterize further the spatial abilities of the rats.

\section{Materials and Methods Methods}

Animals. Thirty-six male Long-Evans rats (University of Lethbridge vivarium) were used. They were housed in pairs in plastic bowls in a laboratory with room temperature maintained at $20-21^{\circ} \mathrm{C}$ and lighted on a $12 / 12 \mathrm{hr}$ light/dark cycle. For 2 weeks after surgery, the rats were taken to the test room, removed from their cages, and handled for about $5 \mathrm{~min}$. At the time the experiment began, the rats weighed $400-500$ gm.

Surgery. Prior to the beginning of the handling procedures, the rats received bilateral cathodal lesions that severed the axons of the fimbriafornix. Cathodal current was passed for $40 \mathrm{sec}$ through 00 stainless steel insect pins insulated with epoxylite except at the surface of their tips. Lesions were made at two sites in each hemisphere using coordinates in reference to bregma and the surface of the dura: $1.3 \mathrm{~mm}$ posterior, $1.5 \mathrm{~mm}$ lateral, and $3.6 \mathrm{~mm}$ ventral, and $1.5 \mathrm{~mm}$ posterior, $0.5 \mathrm{~mm}$ lateral, and $3.3 \mathrm{~mm}$ ventral. Behavioral testing began $15 \mathrm{~d}$ following surgery.

Swimming pool. The swimming pool was located in a test room in which many cues, including windows covered by blinds, counters, cupboards, etc., were present. A $146 \mathrm{~cm}$ diameter and $45 \mathrm{~cm}$ high round white swimming pool was located in the center of the room. It was filled to a depth of $25 \mathrm{~cm}$ with $22^{\circ} \mathrm{C}$ water that was made opaque with the addition of $1500 \mathrm{cc}$ of powdered milk.

Platforms. Depending upon the task, one of two platforms was located in the pool. The visible platform was an $8.5 \mathrm{~cm}^{2}$ wooden block painted black that protruded from the water for $3 \mathrm{~cm}$. A small shelf below the surface of the water served as a step to help the rats climb onto the platform. The hidden platform was made of clear Plexiglas and its $11 \mathrm{~cm}^{2}$ top was located $1 \mathrm{~cm}$ below the surface of the water where it was not visible to a viewer on the surface of the water. The surface of the platform was serrated so that the rats could obtain purchase as they climbed onto it.

Training. For training, the rats were taken to the test room in their home cages. Each pair of rats was then placed in a cage identical to the home cage that was located on a stand near the swimming pool. One of the rats was removed from the cage and placed for $5 \mathrm{sec}$ in the basket of its grid top. The rat then received a trial in the swimming pool after which it was again placed for $10 \mathrm{sec}$ on the top of the holding cage, and then the next trial began. These procedures were used to ensure that the rats were comfortable with the handling and training procedures.

In all of the experiments, starting points for the swims were at the cardinal compass points (N, S, E, and W), which were selected in a semirandom fashion for each rat on each trial. A rat was held by the torso behind the shoulders and gently placed into the water facing the wall of the pool, so its head did not submerge. After its last daily swim, each rat was replaced in its home cage and returned to the colony room.

Videorecording and analysis. On all trials, swim durations, from the time the rat was placed in the water to touching the platform with the forepaws, were timed with a stop watch, the swim path of the rat was drawn on a map of the swimming pool, and the swims were videorecorded. In some tests, the accuracy of a rat's swim was scored on each trial. If a rat swam directly to the platform, staying within an $18 \mathrm{~cm}$ wide corridor from the start point to the platform, then the swim was scored as correct. If a rat deviated from this corridor, it was given an error for that swim (Whishaw, 1985b).

The video camera was used to ensure that the platforms were placed in the same location each day with reference to the pool and room, and the video record subsequently was used for a variety of analyses. For certain tests and probe trials, the rats' swims were digitized using a Peak performance kinematic analysis system (Peak Performance, Denver, CO). Successive video fields (60 fields/sec) were grabbed with a frame grabber and the tip of a rat's nose was manually marked. The swim path and the swimming velocity were then determined using Peak programs and the results, expressed as $\mathrm{x}, \mathrm{y}$-coordinates, were stored in the computer for subsequent averaging, analysis, and display. Since swims took between 1 and $5 \mathrm{sec}$, between 60 and 300 points, were used for determining swim trajectories, distance, and velocity on each trial. (Preliminary digitizing was performed using the automatic features of a number of movement tracking systems; however, the processes by which the automatic systems followed the average exposed surface of the rat did not produce reliable results.)

Histology. At the completion of the experiments, the rats were deeply anesthetized, perfused with saline and saline-formalin, and the brains were removed and stored in a $30 \%$ sucrose formalin solution. The brains were cut in $40 \mu \mathrm{m}$ sections on a cryostat and alternate sections were with cresyl violet and stained for acetylcholinesterase.

\section{Procedure}

Two experiments were performed. In the first experiment, the performance of FF rats and control rats was assessed on a place task that consisted of escaping to a hidden platform using conventional training procedures. In the second experiment, similar groups of rats were first trained to swim to the visible platform and were then evaluated on probe trials to determine whether they attended to distal ambient cues. The same groups were subsequently given a number of additional probe and training tests to confirm that they had learned a place response.

\section{Experiment I: the effect of FF lesions on place learning}

Eleven rats with fimbria-fornix lesions and nine control rats were used. The hidden platform was located in the NE quadrant of the pool. Each rat received two trials each day and each of the two trials began at one of the four start locations, with starting locations changed each day. The trials were given sequentially with an intertrial interval of about $10 \mathrm{sec}$. After the rats had completed 28 trials, the hidden platforn was replaced with the visible platform for six trials. Then, the rats received two more trials with the platform hidden. Trials were videorecorded, swims were recorded on a map of the pool, and latency was recorded with a stop watch.

\section{Experiment II: pretraining on the visible platform followed by probe trials}

Training. Eight rats in each of two groups (FF, control) served in the cue experiment and subsequent testing. In order to gradually adapt the rats to the procedures, training took place in four stages. (1) Each day for $6 \mathrm{~d}$ the rats were placed on the visible platform twice for $30 \mathrm{sec}$ with an intertrial interval of about $10 \mathrm{sec}$. (2) Each day for $4 d$ the rats were placed into the water next to the platform twice, so that they could quickly climb onto it. The rats were placed so that their swims began from the directions of the cardinal compass points, but the rats were released as they faced the platform. After they climbed onto the platform they were left there for $30 \mathrm{sec}$ and intertrial intervals were $10 \mathrm{sec}$. (3) For $2 \mathrm{~d}$ the rats received two swims, one from each of the four start points. For these and subsequent trials they were placed into the water facing the wall. After each swim they were left on the platform for 10 sec and trials were given sequentially with an intertrial interval of 15 sec. (4) On each of $8 d$ the rats received four trials each day, one from each start point, with start points selected in a semirandom sequence. Intertrial intervals were about $10 \mathrm{sec}$. They were left on the platform for $15 \mathrm{sec}$.

Probe tests. At the completion of the training procedures, the rats were given two probe tests. (1) No platform probe: the rats received four swims each day for $4 \mathrm{~d}$, one from each start point. For one of the daily swims (selected semirandomly so that swims from each start point were obtained for each rat), the visible platform was removed from the pool. On these swims, the rats were quickly removed from the water as soon as they left the correct quadrant of the pool, once having entered it. (2) Hidden platform probe: the rats received four swims each day for a second $4 \mathrm{~d}$. On one of the daily swims (chosen semirandomly so that swims from each starting point were obtained for each rat), the visible platform was replaced by the hidden platform. If a rat missed the hidden platform and then swam out of the correct quadrant of the pool, it was quickly removed from the water.

Place training. With the hidden platform located in the same place in which the visible platform had been located, the rats were given four trials each day for $10 \mathrm{~d}$. If a rat failed to find the platform within 60 $\mathrm{sec}$, the rat was removed from the pool, ending that trial.

Matching to place task. The hidden platform was moved to a new location on each day for each of $5 \mathrm{~d}$. The rats received four trials each day, each starting from one of the four start points. They were required to swim until they found the platform on each trial. Measures were latency to find the platform and accuracy. 

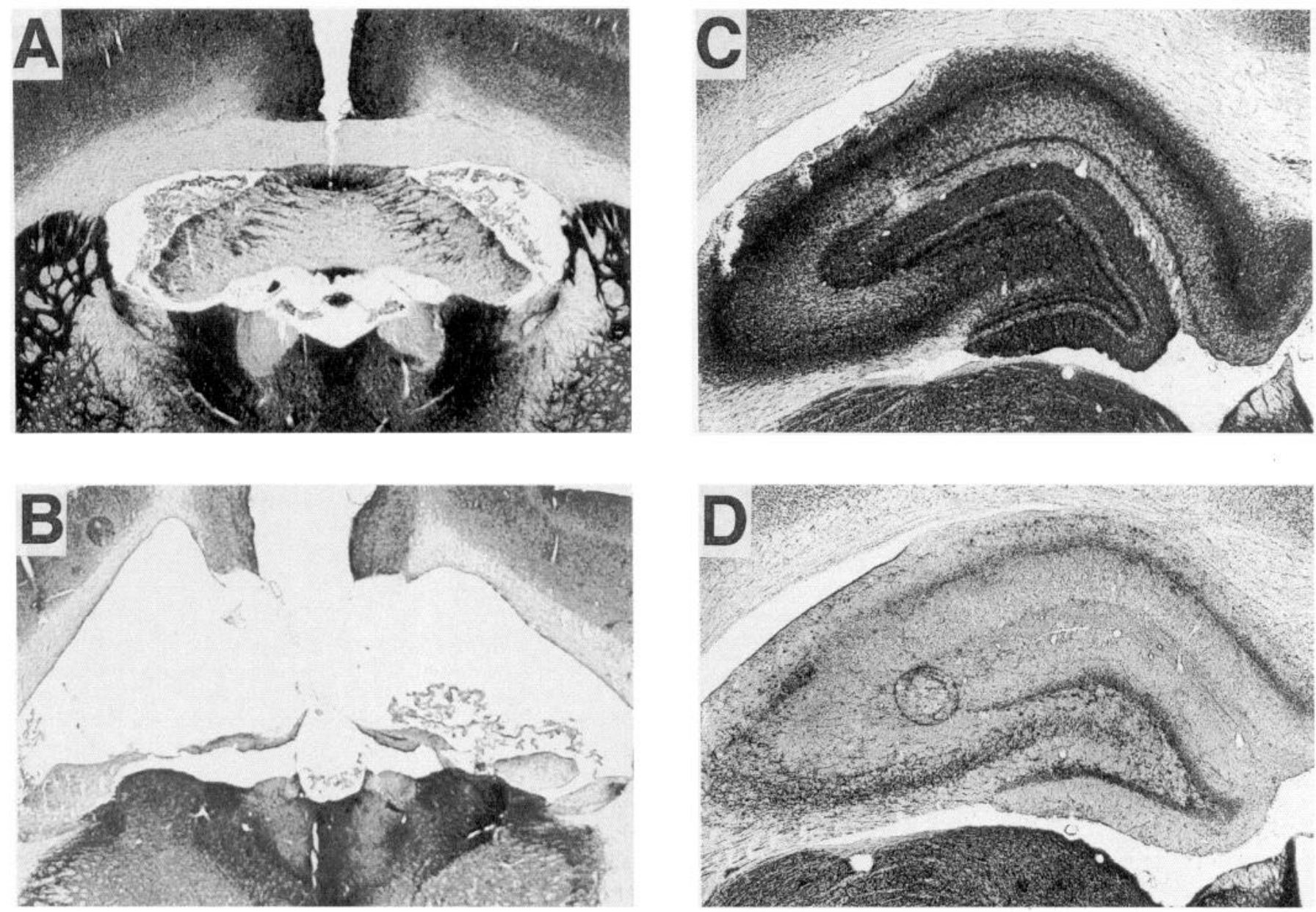

Figure 1. Photomicrographs showing the fimbria-fornix in a control rat $(A)$ and the extent of fimbria-fornix damage in a representative animal in the lesion group $(B)$. Comparisons in $C$ (control) and $D$ (fimbria-fornix) show loss of acetylcholinesterase (AChE) staining in the hippocampus following the fimbria-fornix lesion.

\section{Results}

\section{Histological results}

The dorsal fornix and the fimbria were completely sectioned in all of the rats that were given lesions (Fig. $1 a, b$ ). Some lesions encroached onto the most posterior part of the lateral septal nucleus, the dorsal hippocampal commissure, and the most anterior part of the dorsal hippocampus. The tract made by the electrodes and the lesion only slightly damaged the supracollasal septohippocampal pathways or cortex. Previous work has shown that the supracollasal damage does not produce additional impairments on spatial tasks (Sutherland and Rodriguez, 1989; Jeltsch et al., 1994). Stains for acetylcholinesterase (AChE) revealed extensive depletion of AChE in the hippocampus (Fig. 1c,d). From previous work it is known that the lesion used in the present experiment reduces cholinergic markers by about $70 \%$ in the dorsal hippocampus (Cassel et al., 1991; Jeltsch et al., 1994).

\section{Experiment I: Place experiment}

The pattern of spatial behavior of the control and FF rats was different (Fig. 2). The control rats rapidly learned to swim to the hidden platform, whercas although the rats with FF lesions showed some improvement, they remained severely impaired relative to the control group as indicated by their high mean latencies, $[F(1,18)=20.7, p<0.001]$. When the visible plat- form was substituted for the hidden platform, rats in both groups quickly learned to swim directly to it and there was no group difference $[F(1,18)=0.24, p>0.05]$. When the platforms were again exchanged, the group differences reemerged as the performance of the rats in the fimbria-fornix group deteriorated $[F(1,18)=29, p<0.001]$.

\section{Experiment II: Cue experiment with probe trials}

Acquisition latency. Once the rats were habituated to the room, pool, and platform, they were given 36 trials swimming to the visible platform. There was no difference in the latencies of control and FF rats to reach the visible platform, as animals in both groups uniformly produced swims that were rapid and direct at asymptote $[\mathrm{control}=4.3 \mathrm{sec}$ vs $\mathrm{FF}=4.7 \mathrm{sec}, F(1,14)$ $=0.32, p>0.05]$.

Swim trajectories: visible platform present versus absent. On the last four trials on which the rats swam to the visible platform, the tip of each rat's nose was digitized on each video field and the swim paths were reconstructed (e.g., up to 300 digitized points per swim). These paths are shown in the top portion of Figurc 3. To compare the accuracy of the swim trajectories of the two groups, the digitized curves were interpolated, and the differences between the actual trajectory and the ideal trajectory (the most direct line) were computed for swims from each start- 


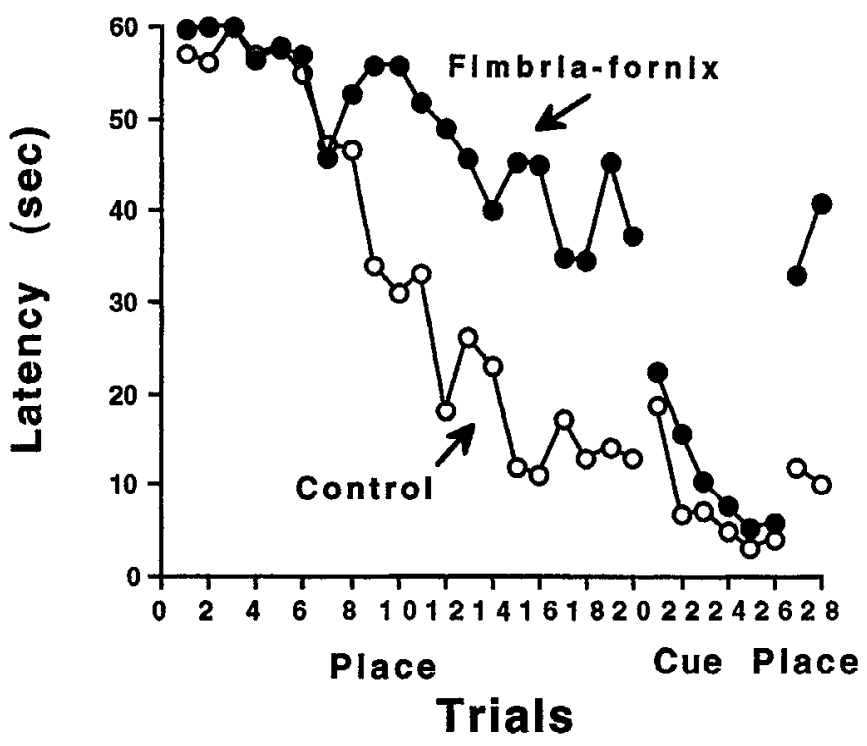

Figure 2. Average swim latencies of control and fimbria-fornix groups on a place task in which the target platform was first hidden at a single location (Place), then visible in that location ( $\mathrm{Cue}$ ), and then again hidden (Place). Note that the fimbria-fornix group displayed almost no learning when the platform was hidden, quickly learned to swim to a visible platform, and then reverted to poor performance when the platform was again hidden. ing position, which provides a difference at each point between the actual and ideal trajectory. ANOVAs performed on these results indicated that there were no group differences in accuracy of swims from any position. The group by position interaction (a comparison of the location of the rats in each group as the swim was performed) was significant for swims from start points 1 and 2 but not from start position 3 and 4 . The significant interaction effects appear due to slightly wider turns at the initiation of swims at the start positions made by the FF group.

The bottom portion of Figure 3 shows the digitized swim paths for the rats on swims on which the platform was removed. ANOVAs performed on the deviations from the idealized swim paths indicated that there was no significant difference for swims taken from start positions 1,2 , and 4 , but there was a significant difference for swims taken from position $3[F(1,14)=5.63, p$ $<0.03]$. Group by swim time interactions were significantly different from start position 1,2 , and $3(p$ s $<0.01)$ but not from start point 4 . Again, the interaction seems mainly attributable to the difference in the width of the turns taken by the two groups at the beginning of the swim.

Average swim path: platform present versus absent. Average swims were obtained by interpolating the digitized records, to equate the number of $\mathrm{x}, \mathrm{y}$-coordinates for each swim for each rat, and then averaging across rats in each group. A swim was considered ended at the point at which a rat came closest to the

\section{Control}
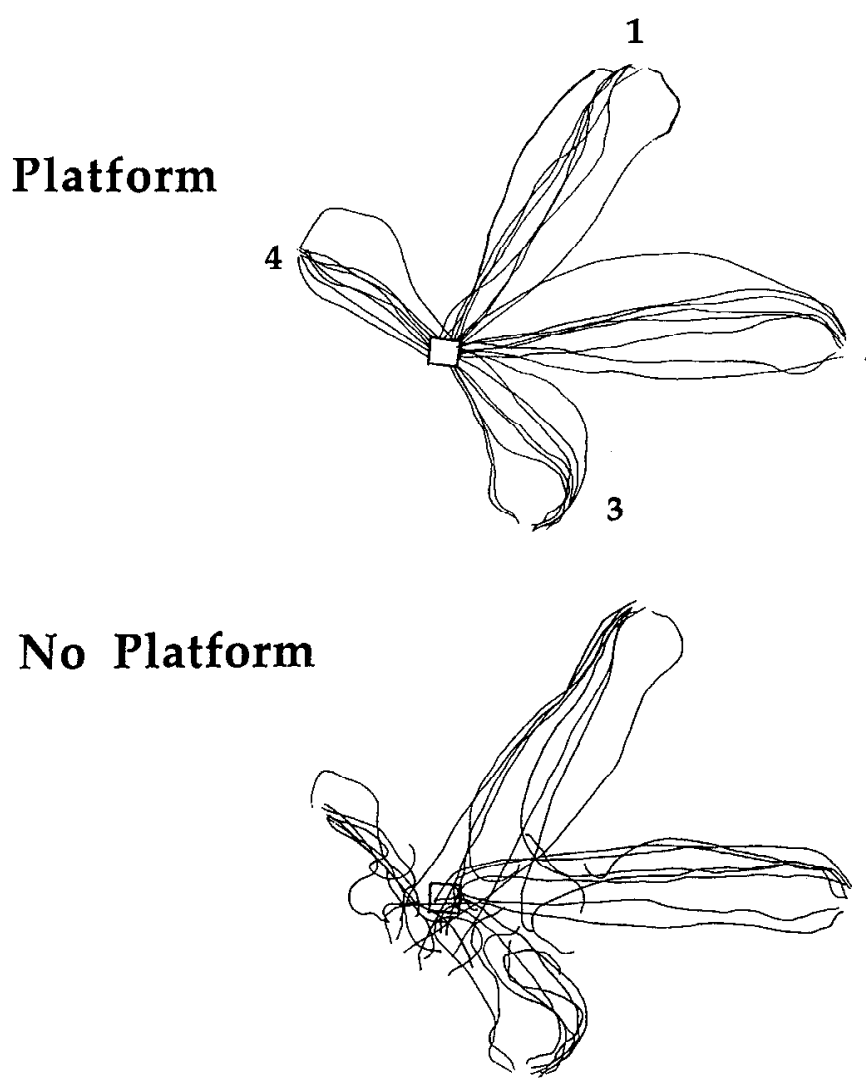

\section{Fimbria/Fornix}
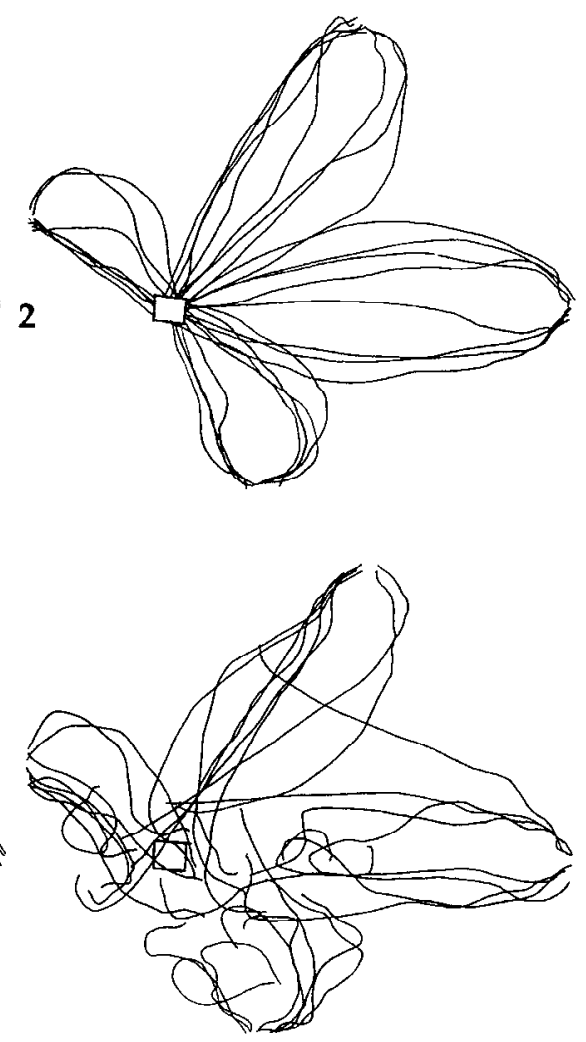

Figure 3. Swim paths of control rats (left) and rats with fimbria-fornix lesions. Top, A platform was present and visible. Note that the swim patterns were similar in both groups, although the fimbria-fornix rats tended to make slightly wider turns when placed into the water facing the wall of the pool. Bottom, No platform was present. Note that both groups of rats swam to the area of the pool in which the platform had been present. The numbers $I 4(\mathrm{~N}, \mathrm{E}, \mathrm{S}, \mathrm{W})$ indicate the order in which start positions were used. Each tracing represents the swim path taken by a rat from that location. 


\section{Control}
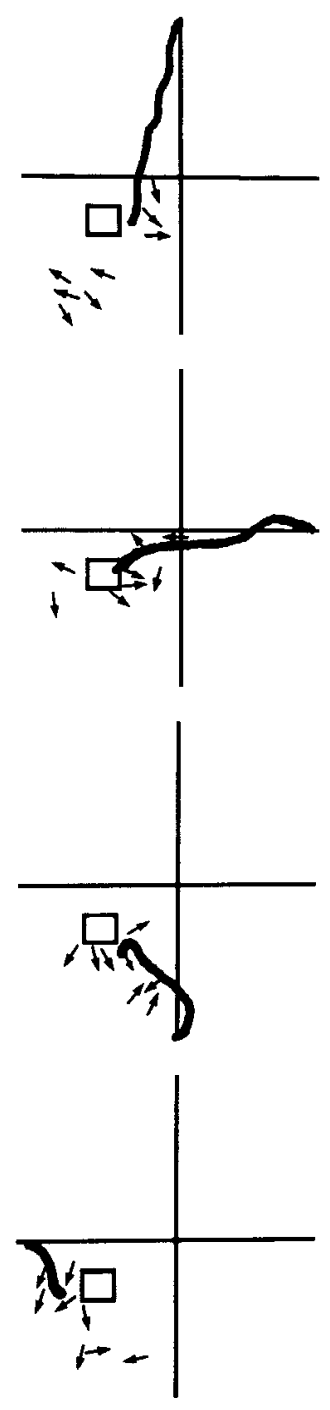

\section{Fimbria/Fornix}
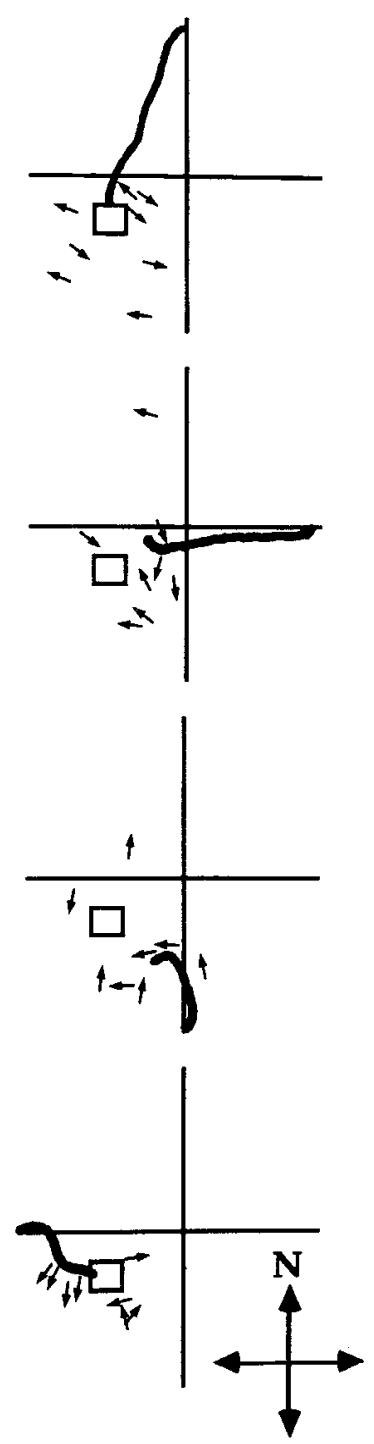

Figure 4. The average swim path of control rats (left) and rats with fimbria-fornix lesions (right) on swims from four start positions (N, E, $\mathrm{S}, \mathrm{W}$, top to bottom) when the platform was not present. The arrows indicate the point at which individual rats terminated their swims by stopping and turning. Note that the average swim for each group leads to the quadrant of the pool in which the visible platform had been located and the rats stopped and turned in close proximity to the platform's previous location.

platform's location. The average swim paths taken by the control and $\mathrm{FF}$ groups when the platform was removed are shown in Figure 4. A comparison of $\mathrm{x}$ - and $\mathrm{y}$-coordinates for swims with the platform present indicated no significant group difference on any of the eight measures (an $x$ and a $y$ value for each of the four swims). When the platform was removed, the $x$ value for position 3 was the only value that gave a significant difference $[F(1,14)=2.68, p<0.05]$.

Figure 4 also shows locations at which rats changed their swimming direction after their swimming course had been set toward the platform. The location and direction of each turn is indicated by an arrow for each rat. These points were characterized by a sharp deceleration in swim speed and an obvious body turn. With the exception of 3 of 32 swims by rats in the FF group and 1 of 32 swims by the control group, all of the directional changes occurred in the quadrant of the pool in which the platform had been located. The average latency to initiate the change in swimming direction was not different between the two groups [control $=2.3 \mathrm{sec}$ vs $\mathrm{FF}=2.38 \mathrm{sec}, F(1,14)=$ $0.03, p=0.86]$. Similarly, the distance from the platform at which the turns were initiated was not significantly different [control $=7.8 \mathrm{~cm}$ vs FF $=8.3 \mathrm{~cm}, F(1,14)=0.21, p=0.42$ ]. It is noteworthy that on some trials ( 9 of 32) several rats in the FF group actually stopped and floated in the vicinity of the platform. (This behavior was also observed in two preliminary studies with five and five rats and eight and eight rats in each group, and provided the incentive to use kinematic measures in the present study.)

Swim velocity: platform present versus absent. Figure 5 (top) shows the average swim velocity to the visible platform from each start location produced by control and FF rats. Typically, velocity was slow as the rats turned away from the wall at the start position and initiated the swim, increased as they swam toward the platform, and decreased as they approached it. The end of the curve is the point at which the rats touched the platform. The shapes of the velocity curves for the two groups are very similar when the platform was present. ANOVAs performed on the interpolated velocity profiles (velocity comparisons at each point along the swim) indicated no significant differences on swims from any start position.

Figure 5 (bottom) shows the average velocity of swims from each start location when the platform was removed. Again, the velocity curves for the two groups are very similar. ANOVAs performed on the normalized velocity profiles indicated that there was no significant group difference on swims from any position. The velocity by swimming time interactions were not significant for any swims. Additionally, the velocity profiles with the platform present and platform absent were very similar, indicating that the rats were decelerating at the same point in both platform present and platform absent conditions.

The most important feature of the velocity profiles in indicating that the rats expected to find a platform at a certain location in the pool is the point at which deceleration of swimming occurred (i.e., the final portions of the velocity curves in Fig. 5). This point occurred in both control and FF groups at about the same time and it occurred in close proximity to the expected location of the platform. There was no significant group difference in the time at which deceleration occurred. This result suggests that control and FF rats expect to reach the platform at its previous location, even though it has been removed.

Probe swims with the hidden platform present. The rats were given 4 further days of training, during which the visible platform was present on three of the trials but replaced by the hidden platform on the remaining trial. For the probe swims when the platform was hidden, if a rat swam out of the correct quadrant without finding the platform, it was removed from the pool. This procedure permitted a direct evaluation of the accuracy of the rats' swims from each of the four starting positions. The control rats found the platform on a total of 24 of 32 trials (four probe trials for each of the eight rats), while the $\mathrm{FF}$ rats found the platform on a total of 23 of 32 trials. The latency to find the platform on successful trials was slightly faster for the control group than it was for the FF group [control $=3.01 \mathrm{sec}$ vs FF $=5.4 \mathrm{sec}, F(1,45)=6.9, p<0.010]$.

Training to swim to the hidden platform. With the platform 


\section{Control}

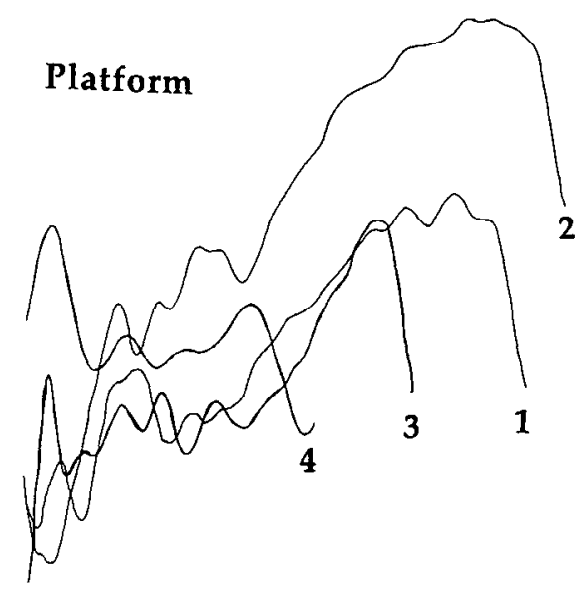

Fimbria/Fornix

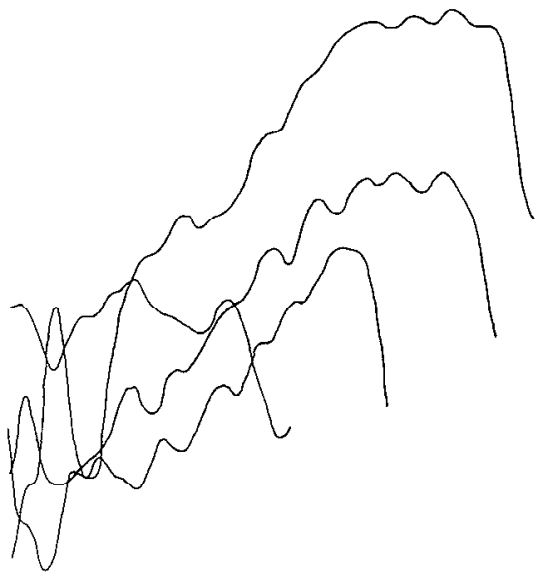

Figure 5. Average swimming velocity of control rats $(l e f t)$ and rats with fimbria-fornix lesions (right) from the four start locations $(1-4 \mathrm{~N}, \mathrm{E}, \mathrm{S}, \mathrm{W})$. Top, A platform was present and visible. Note that the swimming speed increased as the rats turned and swam toward the platform and then abruptly decreased as they came close to it and climbed onto it. Bottom, The platform was not present. Note the similarity in the velocity profiles to those obtained when the platform was present.

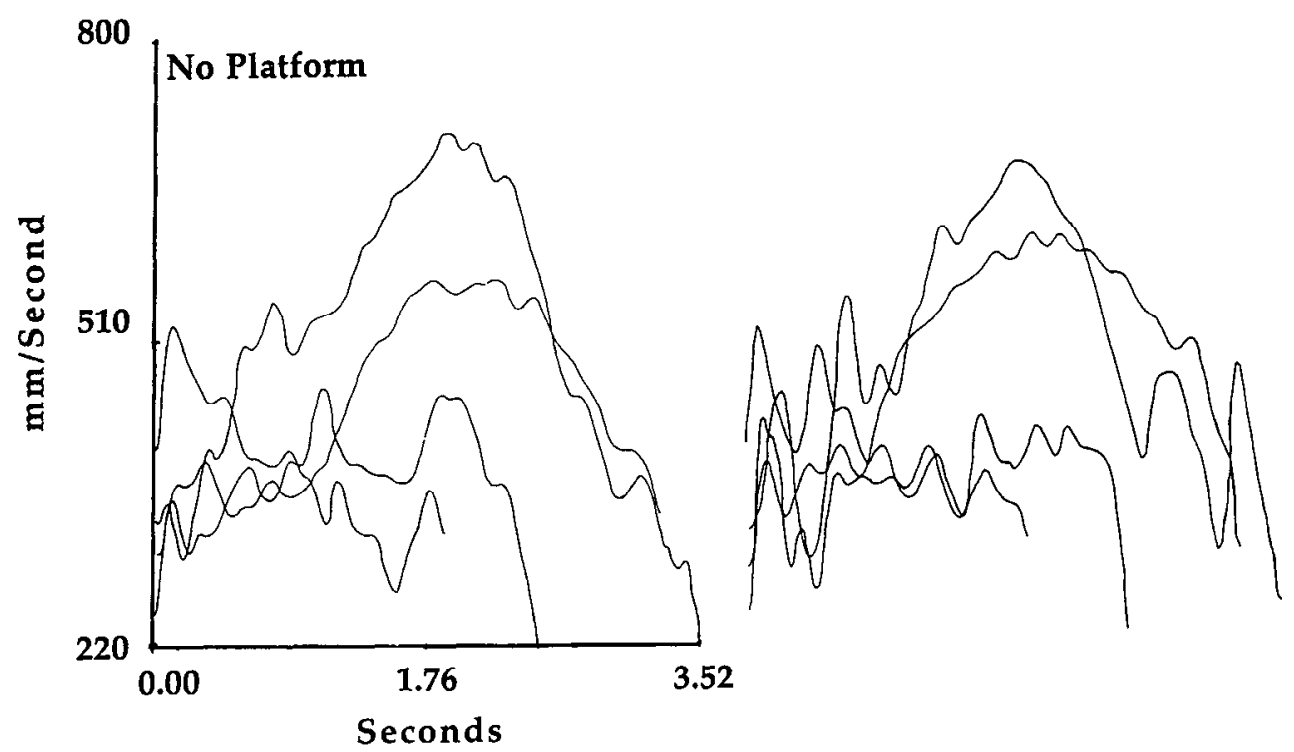

hidden, the rats were given four trials for each of $10 \mathrm{~d}$. Latencies and errors are shown in Figure 6. The latencies of the control group were lower than the latencies of FF group $[F(1,14)=$ $13.9, p<0.01]$, as were errors $[F(1,14)=9.62, p<0.01]$. By the end of the $10 \mathrm{~d}$ of training the performance of the two groups was very similar, however.

Observations of the swimming behavior of the control rats indicated that as they approached the hidden platform their swimming speed slowed, they made lateral movements of the head and they made treading movements with the forepaws. These appeared to be strategies to aid in finding the platform (Sutherland and Dyck, 1984). The swims made by the FF rats were marked by the same features, but the search movements appeared much more exaggerated. As they approached the platform, FF rats slowed and circled or else made large lateral movements. Figure 7 shows representative swims by control and FF rats on the last training trial from position 2 . The exaggerated scarch bchavior of rats in the FF group increased their swim latencies and increased their probability of making an error. Inspection of the records of the rats' swims throughout the $10 \mathrm{~d}$ training period indicated that circling behavior of some of the rats on most trials and by other rats on occasional trials was a major reason that the FF group differed from the control group.

Probe trial after hidden platform training. At the completion of the $10 \mathrm{~d}$ of training, the rats were given a $60 \mathrm{sec}$ probe trial in which the platform was removed. The duration of swimming in each of the four quadrants was measured from the video record and is shown in Figure 8 . The FF group spent significantly less time swimming in quadrant $1[F(1,14)=11.84, p<0.01]$, and significantly more time swimming in quadrant 3 (correct quadrant), $[F(1,14)=7.32, p<0.01]$ than the control group. The finding that the FF group searched the previously correct quadrant more than did the control group may be related to the circling strategies that were displayed during training; i.e., they had been reinforced for circling during training.

Matching to place task. For $5 \mathrm{~d}$ the platform was moved to a new location each day and the rats were given four swimming trials. Figure 9 summarizes the daily latencies and errors made by the control group (left) and the fimbria-fornix group (right) in finding each of the five hidden platform locations. There were significant group, day, and trial differences for both latency and error measures $[F \mathrm{~s}>3$, ps $<0.01]$. The control group reached 


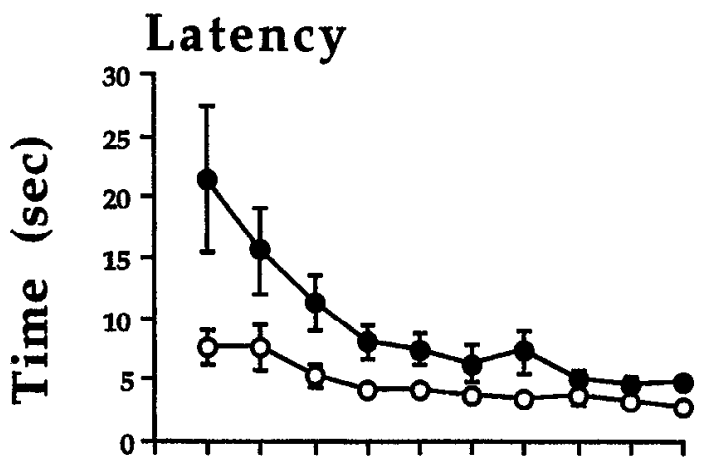

\section{Errors}

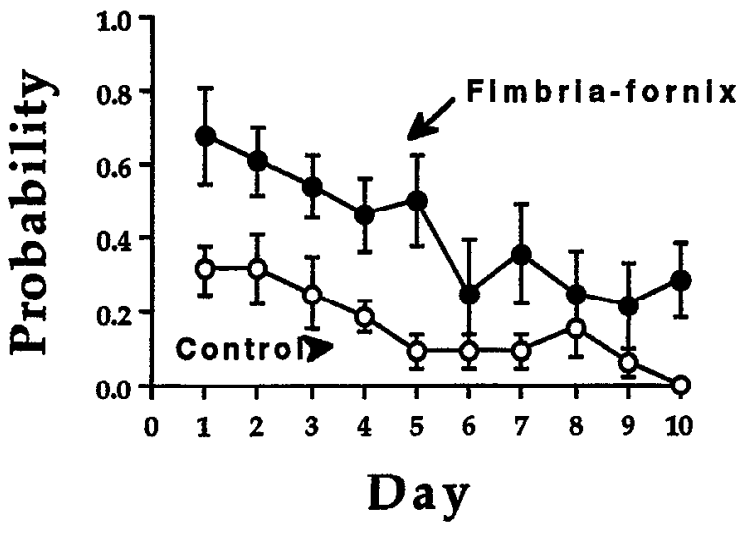

Figure 6. Average swim latency (top) and errors (bottom) made by control rats and rats with fimbria-fornix lesions when trained over $10 \mathrm{~d}$ to find a platform that was hidden below the surface of the water. The location of the platform was the same as that at which it had been when the rats were trained with a visible platform. Note that although the fimbria-fornix group was slower to find the platform and their swims were not as accurate, their performance quickly approximated that of the control rats.

asymptotic performance on the third place position. They searched for the platform at its previous location on the first trial of each day's training before finding it at its new location. Thus, their latencies on the first trials were high. On the second and subsequent trials they swam directly to the new location. Thus,

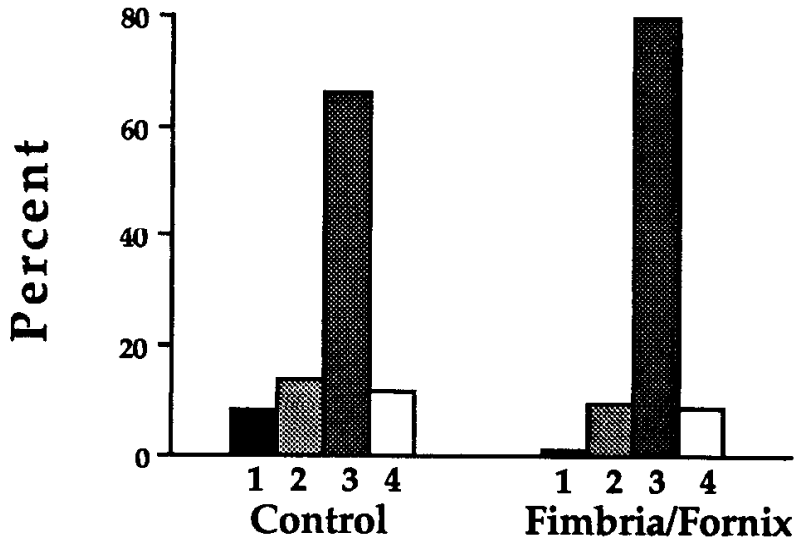

Figure 8. Percentage of swim time spent in different quadrants of the pool on a no-platform probe trial given after training to a hidden platform. The quadrant in which the platform had been located was quadrant 3, and both groups of rats searched predominantly in this quadrant, although the fimbria-fornix rats spent significantly more swimming time in quadrant 3.

on the last three place tasks they learned each new location in "one trial." The rats in the fimbria-fornix group showed significant improvement across trials, but this improvement was rather small and was not characterized by the one-trial learning that was displayed by the control rats. They also displayed no improvement across days. Thus, whereas the control rats had trial 2 latencies that were as low as $5 \mathrm{sec}$ after day 2 and swam almost directly to the platform, the rats in the FF group had trial 2 latencies that exceeded $30 \mathrm{sec}$. At first, they perseverated in swimming in circles in the quadrant in which the visible platform had been located, but thereafter, their swims became haphazard and eventually were characterized by almost random swimming around the pool.

\section{Discussion}

By using a variety of training and measurement procedures we have developed a profile of the impairment displayed by rats with FF lesions in swimming pool spatial tasks. Using a "classical" training procedure in the first experiment, we found that FF rats were extremely impaired in learning to find a platform

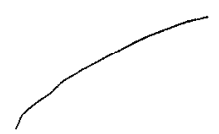

Control
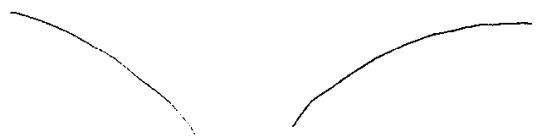

Fimbria/Fornix
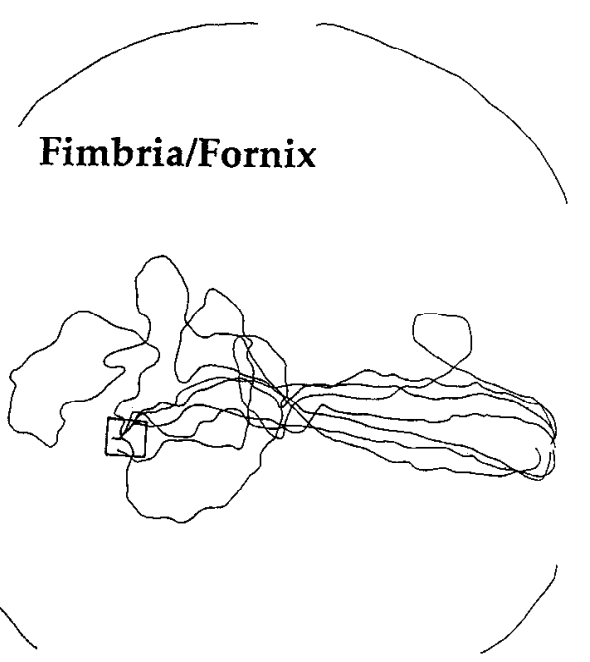

Figure 7. Swim paths made by control (left) and fimbria-fornix rats on the 40th (last) trial of training on the hidden platform task. Note that the swim direction of the fimbria-fornix group approximated that of the control rats but that the FF rats characteristically made circles or loops as they approached the location of the platform. 


\section{Control}
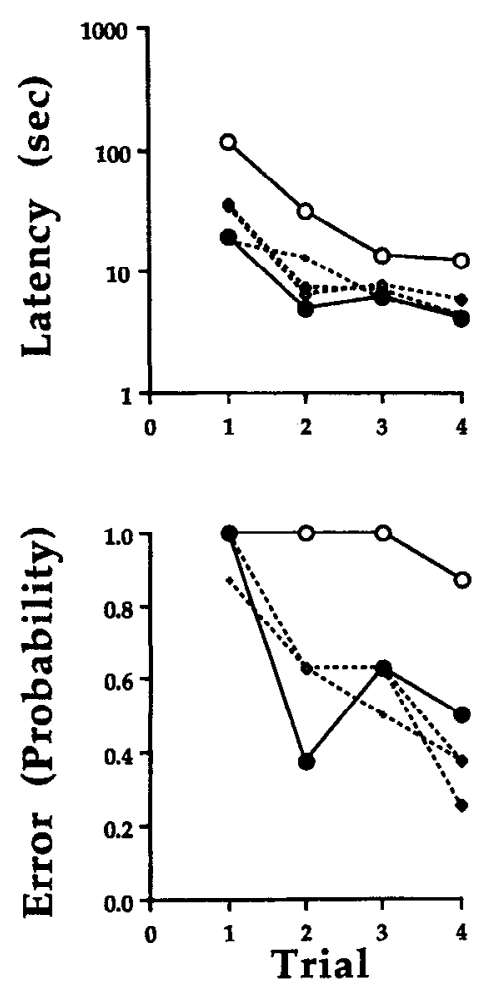

Fimbria/Fornix
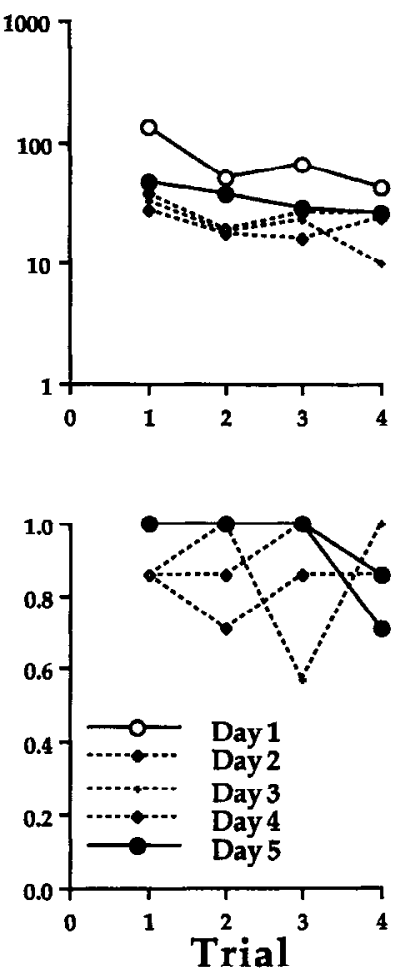

Figure 9. Average swim latencies (semilog plots) and errors across four trials on a task in which the location of a hidden platform changed each day for $5 \mathrm{~d}$. Note that by day 3 the control rats show most improvement in swim latency between trial 1 and trial 2 and were thus showing one-trial learning of the new place tasks. By contrast, the fimbria-fornix rats showed no improvement across days or across trials.

hidden at a fixed location, as they seemingly swam at random until they bumped into it. Although thcy swam directly to a visible platform when it was introduced, this experience was not beneficial as their performance deteriorated when the platforms were again exchanged. In the second experiment, we found that if the rats were first trained to swim to the visible platform, then, when it was removed for probe trials, they swam to that location and searched for the platform. A number of other training and probe procedures confirmed that rats could form a place response. If these rats were then trained to a find the hidden platform at new locations, their performance deteriorated. Thus, the rats were impaired in learning to find the platform, they could learn its location when appropriately cued, but they were again impaired when required to search in a new location. These results suggest that the processes of learning to get to the hidden platform can be dissociated from the processes of knowing where it is.

Results of the first experiment confirm previous studies on rats given FF lesions (Cassel and Kelche, 1989; Sutherland and Rodriguez, 1989), hippocampal lesions (Morris et al., 1982; Sutherland et al., 1982a), and cholinergic muscarinic blockade (Sutherland et al., 1982b; Whishaw, 1985a,b). Since the FF lesions cut the major cholinergic input to the hippocampus as well as other connections (Shute and Lewis, 1963; Lynch et al., 1978; Fibiger, 1982; Mesulam et al., 1983), it is likely that all of these treatments disrupt a neural system involved in spatial behavior. The results of the second experiment find support in other studies that demonstrate that animals with hippocampal dysfunction

do improve on place learning under certain training regimes, including overtraining, pretraining, or training with simplified procedures (Whishaw, 1985a,b, 1989; Sutherland and Arnold, 1987; Whishaw and Petrie, 1988; Eichenbaum et al., 1990; Morris et al., 1990).

Krechevsky (1938) suggests that problems consist of two components: finding the solution and then learning the task. Thus, whereas a common interpretation of the deficits in place learning of rats with hippocampal dysfunction is that they cannot use the relational properties of distal cues to locate a goal (e.g., O'Keefe and Nadel, 1972; Hirsh, 1974; Sutherland and Rudy, 1989; Jarrard, 1993), the rational for the present experiment lay in the recognition that an impairment in place learning of itself cannot indicate whether the animal only fails to use distal cues or cannot use them. Certainly, the behavior of the animals when they are first placed into the swimming pool suggests that they first experiment with various escape possibilities. They scrabble at the wall, swim around its edge, make sorties into its center, and circle, etc. When they discover that the escape platform is at a fixed location, they quickly learn to swim to it (Morris, 1981; Sutherland and Dyck, 1984; Whishaw, 1985a,b; Whishaw and Petrie, 1988). Indeed, Whishaw and Petrie (1988) report that when rats make errors during acquisition they sometimes regress to ineffective escape behaviors. Following this logic, the profile of performance of the rats in the present experiments suggests that they are impaired in using some process necessary for learning how to get to the platform rather than being impaired in learning its location.

To learn to swim directly to a hidden target, an animal must successively shorten the path that is taken and it must progressively improve the heading direction that it takes when the swim is initiated. The ability of animals to make such progressive refinements may be achieved by a process such as path integration (Gallistel, 1990). Path integration is most dramatically displayed by animals that take a circuitous route away from a home base followed by a rapid and direct return. Path integration may be accomplished in one of two ways: by learning to use distal cues to update a position and generate shorter routes or by retaining a record of previous movements from which a shortened trajectory is abstracted. The important aspect of path integration is that it must involve a process of active monitoring/control of ongoing movement to update positions with respect to velocity and line.

The results of the present experiment suggest that it is in some process related to the active monitoring of movements, such as path integration, that the impairment of the FF rats lies. In initially learning a place response they do learn to reach the platform but they do not quickly shorten their swims or develop swims that carry them directly to it. Even after having learned a place response by cuing and additional training, they remain unable to solve new place problems when trained using a classical training procedure. Taken together, these findings suggest that the impairments of the FF rats are most pronounced when they must search for the platform as opposed to swimming to a platform of known location. Another observation that is consistent with the idea that the FF rats' impairment is in a process such as path integration is the observation that even after being cued and well trained, their swim trajectories were not as direct as those of control rats (see also Whishaw, 1985b). How the hippocampus might facilitate place learning through a process such as path integration is not known. Nevertheless, there is ample evidence that the hippocampus is involved in movement: 
its slow wave activity is closely related to movement (Vanderwolf et al., 1973), many of its cells are active in indicating both direction and movement (McNaughton et al., 1983), its stimulation can arrest movement (Bland and Vanderwolf, 1972; Whishaw and Nikkel, 1975), and when it is damaged there are pronounced changes in exploratory and other motoric behaviors (O'Keefe and Nadel, 1978; Amsel, 1993; Whishaw et al., 1994).

There are, of course, other interpretations of the present results. Eichenbaum et al. (1990) suggest that FF rats are capable of using a single room view to guide their swims from one starting position. One could extrapolate to postulate that FF rats can learn to use four separate room views. We think that this possibility is weak if it is considered that in the present study the FF rats that learned the location of the hidden platform were first pretrained with a visible cue and thus had no need to rely on ambient cues. Morris et al. (1990) argue that the hippocampus enhances learning speed and/or motoric competency. Again, we do not think that a learning speed hypothesis easily accounts for the present results as the rats in the present study displayed a place response despite having not been specifically trained to do so. Gabriel et al. (1987) have suggest that the function of the hippocampus is to overcome proactive interference from initially encoded cues. This hypothesis is interesting and consistent with a report by Angeli et al. (1993) that primates can learn one place response but not two. Again, the present results show that the impairment of the FF rats is present both in initial learning and in reversal learning only when they are required to search for the platform. Parsimony suggests that their impairment resides within some aspect of acquisition rather than cue reversal. Finally, despite the remarkable similarity in performance of the control and FF rats in the second experiment, it is possible that the two groups acquire a place response using completely different strategies, for example, a cue-based strategy using a series of rules versus a place strategy. Again, were the $\mathrm{FF}$ rats prone to use conditional strategies one might expect that they would respond solely to the visible cue as opposed to vicariously learning about other ambient cues.

If rats with $\mathrm{FF}$ lesions can learn the location of a platform in relation to ambient cues, as we demonstrate, then this ability must be mediated by some extrahippocampal structure. A number of recent theories postulate that there are many partially independent neural systems for learning and memory (e.g., Packard et al., 1989; Gaffan, 1994). Since swimming pool spatial impairments are obtained following damage to the perirhinal cortex, parietal cortex, or frontal cortex (Kolb et al., 1983), cingulatc cortcx (Sutherland ct al., 1988), subiculum (Morris et al., 1990), and medial striatum (Whishaw et al., 1987; Wiener, 1993; but see Packard et al., 1989) any of these structures may be sufficient for the acquisition of a single place response.

In conclusion, although the function of the hippocampus is still uncertain (Gale et al., 1993; Jarrard, 1993; Horel, 1994; Vanderwolf and Cain, 1994) substantial evidence suggests it is involved in spatial behavior. By using special training, novel kinematic measures, and probe trials, we show that the process of learning to reach a spatial goal or "getting there" can be dissociated from the process of knowing the location of that goal or "knowing where." This finding suggests that impairment of FF rats may reside more in a disability in controlling movement through space rather than in an inability to know the location of places in relation to ambient cues.

\section{References}

Angeli SJ, Murray EA, Mishkin M (1993) Hippocampectomized monkeys can remember one place but not two. Neuropsychologia 31: $1021-1030$.

Amsel A (1993) Hippocampal function in the rat: cognitive mapping or vicarious trial and error. Hippocampus 3:251-256.

Bland BH Vanderwolf CH (1972) Electrical stimulation of the hippocampal formation. Brain Res 43:251-256.

Cassel JC, Kelche C (1989) Scopolamine treatment and fimbria-fornix lesions: mimetic effects on radial maze performance. Physiol Behav 46:347-353

Cassel JC, Kelche C, Peterson GM, Ballough GP, Goepp I, Will B (1991) Graft induced behavioral recovery from subcallosal septohippocampal damage in rats depends on maturity stage of donor tissue. Neuroscience 45:571-586.

Eichenbaum H, Stewart C, Morris RG (1990) Hippocampal representation in place learning. $\mathrm{J}$ Neurosci 10:2531-2542.

Fibiger $\mathrm{HC}(1982)$ ' lhe organization and some projections of cholinergic neurons of the mammalian forebrain. Brain Res Rev 4:327388.

Gabriel M, Sparenborg SP, Stolar N (1987) Hippocampal control of cingulate cortical and anterior thalamic information processing during learning in rabbits. Fxp Brain Res 67:131-152.

Gaffan D (1994) Dissociate effects of perirhinal cortex ablation, fornix transection and amygdalectomy: evidence for multiple memory systems in the primate temporal lobe. Exp Brain Res 99:411-422.

Gale SD, Burr RB, Bigler ED, Blatter D (1993) Fornix degeneration and memory in traumatic brain injury. Brain Res Bull 32:345-349.

Gallistel, CR (1990) The organization of learning. Cambridge, MA: MIT Press.

Hirsh, R (1974) The hippocampus and contextual retrieval of information from memory: a theory. Behav Biol 12:421-424.

Horel JA (1994) Some comments on the special cognitive functions claimed for the hippocampus. Cortex 30:269-280.

Jarrard LE (1993) On the role of the hippocampus in learning and memory in the rat. Behav Neural Biol 60:9-26.

Jeltsch H, Cassel JC, Jackisch R, Neufang B, Green PL, Kelche C, Hertting G, Will B (1994) Lesions of supracallosal or infracallosal hippocampal pathways in the rat: behavioural, neurochemical, and histochemical effects. Behav Neural Biol 62:121-133.

Kolb B, Sutherland RJ, Whishaw IQ (1983) A comparison of the contributions of the frontal and parietal association cortex to spatial localization in rats. J Comp Physiol Psychol 97:13-27.

Krechevsky I (1938) A study of the continuity of the problem solving process. Psychol Rev 45:107-133.

Lynch G, Rose G, Gall C (1978) Anatomical and functional aspects of the septo-hippocampal projections. In: CIBA Foundation symposium 58, Functions of the septo-hippocampal system, pp 5-24. Amsterdam: Elsevier.

McNaughton BL, Barnes CA, O'Keefe J (1983) The contributions of position, direction, and velocity to single unit activity in the hippocampus of freely-moving rats. Exp Brain Res 52:41-49.

Mesulam MM, Mufson EJ, Levey AL, Wainer BH (1983) Cholinergic innervation of cortex by the basal forebrain: cytochemistry and cortical connections of the septal area, diagonal band nuclei, nucleus basalis (substantia innominata) and hypothalamus in rhesus monkey. J Comp Neurol 214:170-197.

Morris RGM (1981) Spatial localization does not require the presence of local cues. I earn Motiv 12:239-260.

Morris RGM, Garrud P, Rawlings J, O'Keefe J (1982) Place navigation impaired in rats with hippocampal lesions. Nature 297:681-683.

Morris RGM, Schenk F, Tweedie F, Jarrard LE (1990) Ibotenate lesions of hippocampus and/or subiculum: dissociating components of allocentric spatial learning. Eur J Neurosci 2:1016-1028.

O'Keefe J; Nadel L (1978) The hippocampus as a cognitive map. Oxford, UK: Clarendon.

Packard MG, Hirsh R, White NM (1989) Differential effects of fornix and caudate nucleus lesions on two radial maze tasks: evidence for multiple memory systems. J Neurosci 9:1465-1472.

Shute CCD, Lewis PR (1963) The ascending cholinergic reticular system: neocortical, olfactory and subcortical projections. Brain 90:497520 .

Sutherland RJ, Arnold KA (1987) Temporally graded loss of place memory after hippocampal damage. Soc Neurosci Abstr S.175. 
Sutherland RJ, Dyck RH (1984) Place navigation by rats in a swimming pool. Can J Psychol 38:322-347.

Sutherland RJ, Rodriguez AJ (1989) The role of the fornix/fimbria and some related subcortical structures in place learning and memory. Behav Brain Res 32:265-277.

Sutherland RJ, Rudy JW (1989) Configural association theory: the role of the hippocampal formation in learning, memory, and amnesia. Psychobiology 17:129-144.

Sutherland RJ, Whishaw IQ, Kolb B (1982a) Spatial mapping: definitive disruption by hippocampal or medial frontal cortical damage in the rat. Neurosci Lett 31:271-276.

Sutherland RJ, Whishaw IQ, Regehr JC (1982b) Cholinergic receptor blockade impairs spatial localization by use of distal cues in the rat. J Comp Physiol Psychol 96:563-573.

Sutherland RJ, Whishaw IQ, Kolb B (1988) Contribution of cingulate cortex to two forms of spatial learning and memory. J Neurosci $8: 1863-1872$

Vanderwolf CH, Cain DP (1994) The behavioral neurobiology of learning and memory: a conceptual reorientation. Brain Res, in press.

Vanderwolf CH, Bland BH, Whishaw IQ (1973) Diencephalic hippocampal and neocortical mechanisms in voluntary movement. In: Efferent organization and the integration of behavior (Masser JD, ed), pp 229-263. New York: Academic.

Whishaw IQ (1985a) Evidence for two types of place navigation in the rat. In: Electrical activity of the archicortex (Buzsaki G, Vanderwolf $\mathrm{CH}$, eds), pp 233-253. Budapest: Hungarian Academy of Sciences.

Whishaw IQ (1985b) Cholinergic receptor blockade in the rat impairs locale but not taxon strategies for place navigation in a swimming pool. Behav Neurosci 99:979-1005.

Whishaw IQ (1985c) Formation of a place learning-set in the rat: a new procedure for neurobehavioral studies. Physiol Behav 35:139143.

Whishaw IQ (1987) Hippocampal granule cell and CA 3-4 lesions impair formation of a place learning-set in the rat and induce reflex epilepsy. Behav Brain Res 24:59-72.

Whishaw IQ (1989) Dissociating performance and learning deficits on spatial navigation tasks in rats subjected to cholinergic muscarinic blockade. Brain Res Bull 23:347-358.

Whishaw IQ, Mittleman G (1986) Visits to, starts, routes and places by rats (Rattus norvegicus) in swimming pool navigation tasks. J Comp Psychol 100:422-431.

Whishaw IQ, Nikkel RW (1975) Anterior hypothalamic electrical stimulation and hippocampal EEG in the rat: suppressed EEG, locomotion, self-stimulation and inhibition of shock avoidance. Behav Biol 13:1-20.

Whishaw IQ, Petrie BF (1988) Cholinergic blockade in the rat impairs strategy selection but not learning and retention of nonspatial visual discrimination problems in a swimming pool. Behav Neurosci 102 662-677.

Whishaw IQ, Tomie J (1987) Cholinergic receptor blockade produces impairments in a sensorimotor subsystem for place navigation in the rat: evidence from sensory, motor, and acquisition tests in a swimming pool. Behav Neurosci 101:603-616.

Whishaw IQ, Mittleman G, Bunch ST, Dunnett SB (1987) Impairments in the acquisition, retention and selection of navigation strategies af ter medial caudate-putamen lesions in rats. Behav Brain Res 24:125138

Whishaw IQ, Casscl J-C, Majchrzak M, Cassel S, Will B (1994) "Short stops" in rats with fimbria-fornix lesions: evidence for change in the mobility gradient. Hippocampus, in press.

Wiener SI (1993) Spatial and behavioural correlates of striatal neurons in rats performing a self initiated navigation task. J Neurosci 13 $3802-3817$. 